\title{
PERSPECTIVE
}

\section{Laser imaging of the retina}

\author{
P F Sharp, A Manivannan, P Vieira, J H Hipwell
}

The development of the scanning laser ophthalmoscope (SLO), first conceived by Webb et al in $1980^{1}$ heralded the first widespread application of lasers for retinal imaging. A highly collimated beam from a laser is swept over the retina delivering all its energy to a very small spot for a very short time, typically of the order of some tens of nanoseconds. Light reflected from the spot is detected and synchronously decoded to form an image on a monitor.

The use of a laser in this way offers a number of fundamental advantages for retinal imaging:

- as the input laser beam occupies only a small part of the pupil most of the pupil is available for light collection permitting non-mydriatic imaging and levels of illumination that are a factor of 200 lower than with the fundus camera

- there is no need to employ high intensity flash so rapid imaging can be carried out offering the potential for serial imaging

- since imaging is carried out point by point, data are readily acquired as digital images allowing quantitative measurements and image processing

- since the detected light is focused at a single point confocal imaging can be employed to give three dimensional images

- the scattered light can be imaged separately from the reflected component, Tyndall imaging, to enhance pathological structures that have a high scattering component

- the wavelength of the illuminating light can be selected by choice of laser so offering the potential for multispectral imaging

- changes in polarisation of the reflected light can be used to measure retinal nerve fibre layer (RNFL) thickness.

While a number of these factors make imaging simpler there are three major advances that the SLO offers to retinal imaging - the ability to do quantitative imaging, multispectral imaging, and three dimensional imaging.

\section{Quantitative imaging}

The SLO is inherently a digital imaging technique; the image is produced by taking a series of samples of the light reflected from the retina as the beam is scanned in a raster fashion whereas with the fundus camera the whole of the retina is imaged simultaneously. However, the advantage of digital acquisition of data is now available with the digital fundus camera, so what advantages does laser imaging offer?

One of the hopes of digital imaging is the automated analysis of retinal images both to identify abnormal pathology and to quantify the degree of pathology present. ${ }^{2}$ The precursor to image analysis is the need to correct for any non-uniformities in the illumination of the retina. ${ }^{3}$ In theory the SLO provides a uniform illumination of the retina so minimising the need for correction. In practice, however, there is still some non-uniformity in illumination.

A second advantage is the ability to select the laser wavelength to improve the contrast of the features under analy- sis. This will be discussed later, although relatively little work has been done on it.

But perhaps the main contribution is the ability of the SLO to image rapidly. Since there is no need to utilise flash, images can be taken rapidly, as one avoids the problem of the slow flash recharging time, and the consequent lower light levels mean that the patient is also able to tolerate a long sequence of images. Initially such studies were done by video recording fluorescein angiograms and digitising them off line. ${ }^{4}$ However, such a process leads to a significant degradation in image quality. With modern PCs and frame grabbers, it is possible to image at frame rates of 25 frames per second for time sequences of up to 25 minutes.

This approach gave the possibility of quantifying the passage of fluorescein dye through the retina and relating this to blood flow. The hope was that subtle changes in the retinal circulation, such as those resulting from a deviation of the viscosity of blood from the norm, could be followed closely. This might enable the onset of retinopathy to be anticipated and allow the progress of any treatment to be accurately measured. While the technical problems can be overcome, measurement of blood flow is still hampered by the need to measure vascular volume. Attempts to do so $^{5}$ have been based on the assumption that this parameter is directly related to the diameters of the arteries and veins supplying and draining the relevant quadrant. Also it must be assumed that the flow of fluorescein relates directly to the flow of blood, which may be invalid for pathological cases.

Hipwell and colleagues ${ }^{6}$ have demonstrated the potential value of a digital SLO in quantifying the state of the retinal circulation. They measured the curves showing fluorescence as a function of time after injection at every point in the image. They then parameterised the curves reporting that the parameter showing time to reach maximum fluorescence was the most informative (Fig 1). While the

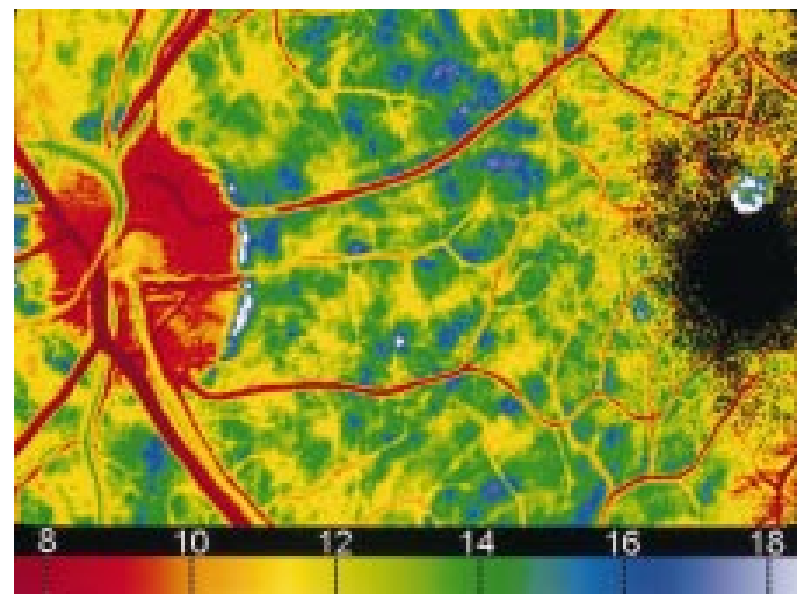

Figure 1 Parametric image in which the colour shows the time (in seconds) for the fluorescence to reach its maximum value. The patient has mild diabetic retinopathy. 
technical requirements for acquiring the data can readily be met in the clinical environment, to achieve a quantitative analysis of the dynamics of fluorescein requires images to be precisely aligned to take account of eye movement. Currently this process, while it can be largely automated, is very time consuming.

Rather than measure fluorescein flow, the SLO has also been used to study directly blood cell dynamics. Blood cells are labelled with a fluorescent label in vitro and readministered. The flow of labelled cells through the vasculature can then be studied. Several methods have been proposed to study leucocyte dynamics in vivo. The problem is to achieve a good labelling while ensuring the viability of the cells. Hossain and colleagues ${ }^{7}$ have developed a method for labelling leucocytes with a non-toxic fluorescent marker that not only preserves cell viability but is also a marker of viability. Using a digital SLO they have measured the velocity of cells and number per unit area.

Such work will not only further our understanding of cell kinetics in vivo but also the effect of disease processes and the influence of treatment. While to date such studies have been restricted to animals, in future it should be possible to perform them in humans.

\section{Multispectral imaging}

Laser imaging of the retina offers the ability to interrogate retinal tissue with light of very specific wavelength. This offers the potential of detecting subtle changes in the function of retinal tissue in response to disease. The goal is that by comparing images produced at a number of different wavelengths, an in vivo spectral analysis of the retina can be performed. Multispectral imaging has been performed with a conventional fundus camera by using filters. This has the obvious disadvantage of reducing the intensity of the illuminating beam. Nevertheless, Delori et $a l^{8}$ and Ducrey et $a l,{ }^{9}$ using a modified fundus camera and interference filters, showed that anatomical and pathological details in the ocular fundus could be seen with increased contrast with monochromatic illumination of appropriate wavelengths instead of white light. For example, arteries and veins are best differentiated from each other around $600 \mathrm{~nm},{ }^{8}$ haemorrhages and microaneurysms were reported to be best seen in the $525-575 \mathrm{~nm}$ region, whereas choroidal folds, melanoma, and naevus were seen better in the $600-650 \mathrm{~nm}$ region. ${ }^{9}$ Using similar methods, degenerative changes in the retinal vessels were studied by Kaefer ${ }^{10}$ and human albinism by Abadi and Dickinson. ${ }^{11}$

In comparison, laser light has a narrow spectral range, effectively offering monochromatic imaging. Present commercial SLOs are available with a choice of wavelengths; commonly argon blue (488 nm), green (514 nm), He-Ne $(633 \mathrm{~nm})$, visible diode lasers $(630-670 \mathrm{~nm})$, and near infrared lasers (780-830 $\mathrm{nm})$.

An SLO employing a specific wavelength has been used to enhance pathological features; macular lesions in $\mathrm{X}$ linked recessive retinoschisis, ${ }^{12}$ papilloedema in idiopathic intracranial hypertension. ${ }^{13}$ Remky et al ${ }^{14}$ compared the effect of different wavelengths on angioscotometry.

Of course, altering the wavelength also changes the degree to which the light will penetrate tissue. In general, shorter wavelengths such as argon $(488 \mathrm{~nm})$ are useful for fluorescein angiography studies ${ }^{6} 15$ observing patterns of herpes simplex keratitis ${ }^{16}$ and leucocyte velocity. ${ }^{77}$ Green $(514 \mathrm{~nm})$ has been used for densitometry study. ${ }^{18}$

Longer wavelengths such as near infrared $830 \mathrm{~nm}$ are useful for indocyanine angiography, ${ }^{19}$ choroidal abnormalities such as naevi, ${ }^{20}$ imaging through lens opacities, ${ }^{21}$ macular oedema and holes, ${ }^{22}$ and drusen. ${ }^{23}{ }^{24}$ Freeman et $a l^{25}$ reported simultaneous indocyanine green and fluorescein angiography using a confocal scanning laser ophthalmoscope.

True colour fundus imaging with a scanning laser ophthalmoscope is possible by sequentially imaging the fundus using blue, green, and red lasers. ${ }^{26}$ Colour SLO imaging has the obvious advantage of creating a colour view of the fundus with which clinicians are most familiar and for differentiating various pathological conditions such as cotton wool spots and drusen which have a similar colour. Some image processing techniques have been applied to colour fundus images specifically to take advantage of this colour information. ${ }^{27} 28$

We have now developed a true colour digital imaging system by using red, green, and blue lasers in a fast pulse mode giving live colour images at the same rate as a monochrome system and which could be either recorded on a VHS video tape or captured as a digital images. Our preliminary work shows images from a diabetic patient (Fig 2).

Perhaps the holy grail of multispectral imaging is to produce images of tissue function. Functional diagnosis of the metabolism of the human ocular fundus is possible by determining the oxygen saturation in the blood. Determination of perfusion of tissue-for example, ischaemia, could be performed by spectroscopic analysis of retinal tissue by measuring the amount of light reflected at three different wavelengths. ${ }^{29}$ While this is still to be achieved, the monitoring of changes in treatment of diabetic diseases has been reported by measuring the optical reflectance of hard exudates. ${ }^{30} 31$

The fundus exhibits autofluorescence when excited with $450-500 \mathrm{~nm}$ light, emitting light with a peak between 520 $600 \mathrm{~nm}$. The autofluorescence is mainly due to lipofuscin in the retinal pigment epithelium (RPE). Von Ruckmann et $a l^{32}$ described a technique of in vivo imaging of autofluorescence of the fundus using a scanning laser ophthalmoscope.

They reported reduced autofluorescence or hypofluorescence in the foveal regions caused by retinal xanthophyll and areas with retinal atrophy such as age related macular disease and autosomal dominant retinitis pigmentosa. Increased autofluorescence or hyperfluorescence was reported ${ }^{33}$ in some genetically determined retinal diseases such as Best's disease, macular dystrophies, and fundus flavimaculatus as a result of diffuse accumulation of lipofuscin in the pigment epithelium.

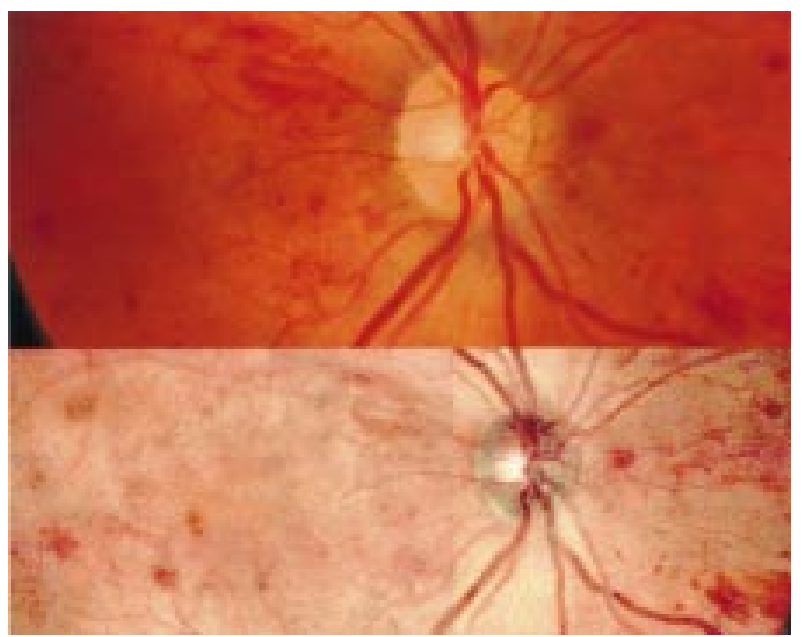

Figure 2 Upper image is a conventional fundus camera image of a patient with mild diabetic retinopathy. The lower image is the colour SLO picture of the same patient. Note that for the colour SLO image two separate images have been combined to give the requisite field of view. 


\section{Three dimensional imaging}

The most popular technique currently available for three dimensional imaging of the retina is the confocal scanning laser ophthalmoscope (cSLO). ${ }^{34}$ The technique is based on the collection of a sequence of images (between 32 and 50) of the retina, each one at a different depth, the depth being selected by the position of a confocal aperture positioned in front of the detector. From this set it is possible to build an axial profile of pixel intensity versus plane depth for each pixel of the image, by considering the value of the intensity of all the corresponding pixels in all the images. From the analysis of the shape of the axial profile, the tomographic information about the retina is obtained.

The shape of the profile will depend upon the amount of light reflected; thus any highly reflecting layer will be identified as a peak on the profile. The most common way to measure the topography of the retina is by calculating the centre of gravity of the axial profile and considering it as the depth of the most reflective layer. ${ }^{35}$ This approach is valid only if the retina is considered to have a single reflective layer in which case the axial profile has a perfect Gaussian shape. However, if a red or infrared laser is being used then owing to the high penetration into the retina tissue, ${ }^{36}$ the reflection from more than one layer may be present and the axial profile will be asymmetric.

To overcome the problem of the asymmetry, more sophisticated algorithms have been developed to fit a Gaussian shape by considering only the part of the axial profile corresponding to the strongest reflector ${ }^{37} 38$ and discarding all the information from the other reflectors. Alternatively it can be assumed that the retina has more than one reflective layer and do the fitting with more than one Gaussian shape. $^{22}$

Independent of the technique used to find the depth of the main reflector, it is possible to build a topographic representation of the retina (Fig 3), and it is on this analysis that most of the current applications of the cSLO are based. One of the most popular applications is the measurement of different topographic parameters of the optic cup with the aim of detecting the presence of glaucoma. ${ }^{39} 40$ Some papers seem to show that the technique could be promising in this field but there was always the problem of a relative high variability in the measurements of the different tomographic parameters.

The reasons why the variability of tomographic measurements is relatively high was studied by a number of authors. ${ }^{41-43}$ The main reasons cited include the movement of the eye during measurement, changes in the position of the patient's head on different measurements, and error introduced by the operator when defining the region of interest on the fundus inside which the volume should be calculated. The first two are difficult to quantify but the last one can be estimated by considering a circular area of interest that surrounds the optic cup; an error of a single

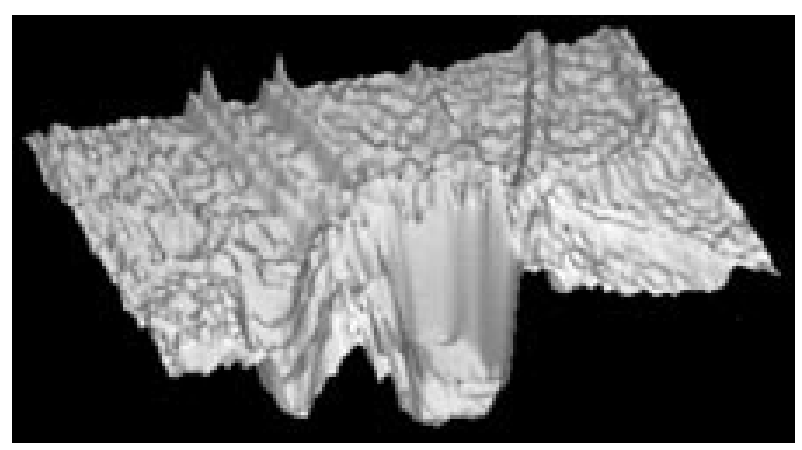

Figure 3 Three dimensional rendered topographic reconstruction of the retina through the optic disc. pixel in the position of the centre of the area will imply a variation of $2.45 \%$ and the error of 10 pixels a variation of $32.2 \%{ }^{44}$ To obtain good reproducibility for the topographic measurements of the retina, a method independent of the user needs to be found.

Some examples of other applications of the cSLO that rely on the topographic measurement of some variables are-optic disc tomography, ${ }^{45}{ }^{46}$ macular holes, ${ }^{47}$ macular oedema, ${ }^{48}$ and monitoring papilloedema in idiopathic intercranial hypertension. ${ }^{49}$

With the cSLO it is possible not only to measure elevation and volume but also to estimate retinal thickness. Considering that the two main reflectors of the retina are the internal limiting membrane/RNFL and the RPE, the distance between them is a good indicator of the retinal thickness. If the axial profile is fitted with two Gaussian shapes, each will characterise one of the reflectors, and so it is possible to calculate the distance between them. Alternatively, if the two reflective layers cannot be discriminated in the axial profile, some information of the retinal thickness can still be found by measuring the full width half height of the axial profile. ${ }^{22} 50$ Figure 4 shows the thickness information of the fundus of a patient with macular hole and surrounding cuff of subretinal fluid (white represents thicker structures and black thinner ones). It is clear that the increase of thickness is due to the presence of fluid.

The thickness of the NFL can also be measured using scanning laser polarimetry by modifying the traditional scanning laser ophthalmoscope. ${ }^{51}$ This technique uses the fact that the NFL has birefringent properties that change the characteristics of a highly polarised laser beam when it passes through it. By analysing this change of polarisation it is possible to determine the thickness of the nerve fibre layer. The technique was introduced commercially in 1993, and has been applied to the detection of glaucoma, ${ }^{52}$ the effect of aging on the thickness of the RPE, ${ }^{53}$ and the study of ocular hypertension. ${ }^{54}$ There are also studies showing good reproducibility of the technique if the same operator is used, and worse if more then one operator is being compared. ${ }^{55} 56$

One of the major limitations of the cSLO technique is its axial resolution which is constrained by the optics of the eye. The theoretical limit is around $300 \mu \mathrm{m}$ for a wavelength of $633 \mathrm{~nm},{ }^{57}$ which is relatively large compared with the thickness of the retina. It is important to address this limitation and, as is done in confocal microscopy, mathematical deconvolution provides a possible answer. ${ }^{58}$ Different deconvolution algorithms are available, each can be used requiring different levels of information about the noise characteristics and blur function of the cSLO.

The confocal aperture in the cSLO that allows the depth discrimination also offers the potential for reducing the amount of scattered light detected, increasing the contrast of the images. It is also possible to position the confocal aperture so as to collect only the scattered light, the so called indirect, Tyndall or dark field image. ${ }^{34}$

The indirect mode image can produce very interesting results because it allows the detection of structures that would otherwise be very difficult to see-for example, drusen. ${ }^{24}$ Recently ${ }^{59}$ it has been reported that the indirect mode can be used to detect macular oedema. Our measurements have also shown that macular oedema can be detected sometimes using the indirect mode. An example of a indirect image obtained from a patient with macular hole is shown in Figure 4 where it is possible to see a rough surface around the macular hole, caused by the presence of fluid.

Recently a new technique for tomographic analysis of the retina has been commercialised, optic coherence 


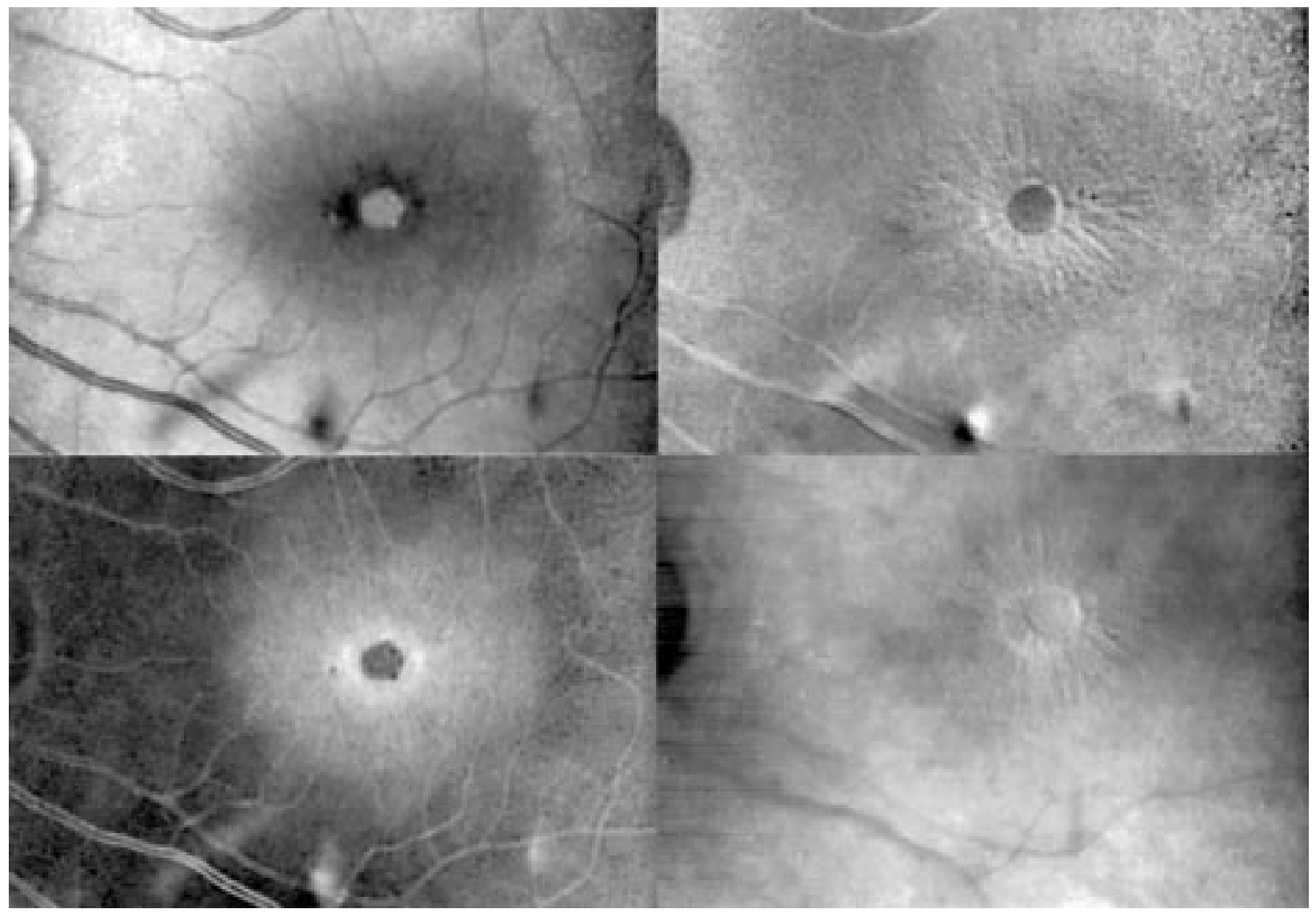

Figure 4 Patient with a macular hole. The top left is an SLO image of the fundus using an infrared laser; the top right shows a topographic image in which white indicates elevated areas; bottom left is a thickness image in which white indicates thicker areas; and bottom right is an indirect mode image.

tomography (OCT). ${ }^{60}$ This technique is similar to the B scan mode of the ultrasound but using near infrared light instead of sound. While pulsed lasers have been used, superluminescent diodes are now the light source of choice. In it the light reflected by retinal layers at different depths will have different times of flight that are found by using low coherence interferometry. The advantage of this procedure is that the optics of the eye do not limit the resolution and $10 \mu \mathrm{m}$ axial resolution has been claimed. These devices do a single line profile through the retina at one time, taking around 2.5 seconds for each profile.

The reproducibility of the OCT has been reported to be good $^{6162}$ but is dependent on good eye fixation and the type of algorithms used. Because of the high resolution of the technique, it has been applied to many retinal pathologies such as macular oedema, ${ }^{63}$ drusen, ${ }^{64}$ glaucoma, ${ }^{65}$ optic pit, ${ }^{66}$ and age related macular degeneration. ${ }^{67}$

\section{The future}

The SLO has been available for routine ophthalmic imaging for over 10 years, yet it has only found application in a relatively small number of departments and often in a very specialised form. As a device for routine imaging it suffers from the disadvantage of not only being technically more complex than a fundus camera, but also may have a smaller field of view than the fundus camera, although one device has recently been announced with a field of view of $180^{\circ}$.

This paper has discussed three areas in which the SLO has particular strengths which may yet offer it a role in the clinic. Three dimensional imaging of the retina has obvious clinical value but the fundamental limitation in slice width will make it a less attractive option than OCT for precise quantitation. While much has been published on the value of the SLO for assessing cup volume, we believe that its value is limited, both from the inherent accuracy of its measurements and the difficulty in defining the cup boundaries. However, there are a number of other pathologies for which it may prove useful but further clinical work is required.

The ability to perform rapid imaging, particularly of the dynamics of fluorescein, is potentially of great clinical interest, although the problem of achieving image registration has still to be addressed. The study of labelled cells in humans may have greater physiological relevance than fluorescein dynamics although is more likely to remain a research tool.

While the study of blood flow and cell kinetics is important, what would probably have the greatest clinical impact would be the ability to produce images of retinal tissue function, in particular perfusion. Multispectral imaging offers a possible route to that goal although there are still many problems to be addressed. The recently developed technique of true colour imaging of the retina may represent the first step in that process.

The authors would like to acknowledge the assistance of Professor John Forrester, Department of Ophthalmology, University of Aberdeen, Dr John Olson, the Eye Clinic, Dr Chea Lim and Dr K McHardy, the Diabetic Clinic, Grampian University Hospital.

P F SHARP A MANIVANNAN P VIEIRA

J H HIPWELL

Department of Biomedical Physics and Bioengineering, University of Aberdeen

Correspondence to: Professor P F Sharp, Department of Biomedical Physics and Bioengineering, University of Aberdeen, Foresterhill, Aberdeen AB25 2ZD 
1 Webb RH, Hughes GW, Pomerantzeff O. Flying spot TV ophthalmoscope. Appl Opt 1980;19:2991-7.

2 Phillips RP, Spencer T, Ross PGB, et al. Quantification of diabetic maculopathy by digital imaging of the fundus. Eye 1991;5:130-7.

Cree MJ, Olson JA, McHardy MC, et al. The preprocessing of retinal images for the detection of fluorescein leakage. Phys Med Biol 1999; 44:293-308.

4 Wolf S, Toonen H, Koyama T, et al. Scanning laser ophthalmoscopy for the quantification of retinal blood-flow parameters: a new technique. In: Nasemann JE, Burk ROW, eds. Scanning laser ophthalmoscopy and tomography. Munich: Quintessenz, 1990:91-6.

5 Bursell S-E, Clermont AC, Kinsley BT, et al. Retinal blood flow changes in patients with insulin-dependent diabetes mellitus and no diabetic retinopathy: a video fluorescein angiography study. Invest Ophthalmol Vis Sci 1996;37:886-97.

6 Hipwell JH, Manivannan A, Vieira P, et al. Quantifying changes in retinal circulation: the generation of parametric images from fluorescein angiograms. Physiol Meas 1998;19:165-80.

7 Hossain P, Liversidge J, Cree MJ, et al. In vivo cell tracking by scanning laser ophthalmoscopy: quantification of leukocyte kinetics. Invest Ophthalmol Vis ophthalmoscopy: quantif

8 Delori FC, Gragoudas ES, Francisco R, et al. Monochromatic ophthalmoscopy and fundus photography: the normal fundus. Arch Ophthalmol 1977;

9 Ducrey NM, Delori FC, Gragoudas ES. Monochromatic ophthalmoscopy and fundus photography: the pathologic fundus. Arch Ophthalmol 1979;97: 288-93.

10 Kaefer O. Degenerative changes in retinal vessels: photodocumentation with monochromatic filters. Arch Ophthalmol 1980;98:303-6.

11 Abadi RV, Dickinson CM. Monochromatic fundus photography of human albinos. Arch Ophthalmol 1983;101:1706-11.

12 Yamaguchi K, Satoh T, Takahashi S. Observation of macular lesion in $\mathrm{X}$-linked recessive retinoschisis by scanning laser ophthalmoscope. Ann Ophthalmol Glaucoma 1998;30:348-50.

13 Mulholland DA, Craig JJ, Rankin SJA. Use of scanning laser ophthalmoscopy to monitor papilloedema in idiopathic intracranial hypertension. $\mathrm{Br} \mathcal{F}$ Ophthalmol 1998;82:1301-5.

14 Remky A, Beausencourt E, Elsner AE. Angioscotometry with the scanning laser ophthalmoscope: comparison of the effect of different wavelengths. Invest Ophthalmol Vis Sci 1996;37:2350-5.

15 Howe LJ, Stanford MR, Whiston R, et al. Angiographic abnormalities of experimental autoimmune uveoretinitis. Curr Eye Res 1996;15:1149-55.

16 Tanaka T. Early pathologic patterns of herpes simplex keratitis detected by scanning laser ophthalmoscope. fpn f Clin Ophthalmol 1998;52:1605-10.

17 Kinukawa Y, Shimura M, Tamai M. Quantifying leukocyte dynamics and plugging in retinal microcirculation of streptozotosin-induced diabetic rats. Curr Eye Res 1999;18:49-55.

18 Van Norren D, Van De Kraats J. Imaging retinal densitometry with a confocal scanning laser ophthalmoscope. Vis Res 1989;29:1825-30.

19 Schneider U, Sherif-Adel S, Gelisken F, et al. Detection of choroidal aneurysms with indocyanine green videoangiography. Graefes Arch Clin Exp Ophthalmol 1998;236:193-5.

20 Takamine Y, Shiraki K, Kohno T, et al. Visualizing choroidal nevi by monochromatic light with a scanning laser ophthalmoscope. Folia Ophthalmol fap 1997;48:86-90.

21 Kirkpatrick JNP, Manivannan A, Gupta AK, et al. Fundus imaging in patients with cataract-role for a variable wavelength scanning laser patients with cataract-role for a variable wa

22 Vieira P, Manivannan A, Lim CS, et al. Tomographic reconstruction of the retina using a confocal scanning laser ophthalmoscope. Physiol Meas 1999;

23 Haynes RJ, Manivannan A, Walker S, et al. Imaging of optic nerve head drusen with the scanning laser ophthalmoscope. Br F Ophthalmol 1997;81: 654-7.

24 Sharp PF, Manivannan A. The scanning laser ophthalmoscope. Phys Med Biol 1997;42:951-66.

25 Freeman WR, Bartsch DU, Mueller AJ, et al. Simultaneous indocyanine green and fluorescein angiography using a confocal scanning laser ophthalmoscope. Arch Ophthalmol 1998;116: 455-63.

26 Manivannan A, Kirkpatrick JNP, Sharp PF, et al. Novel approach towards colour imaging using a scanning laser ophthalmoscope. Br f Ophthalmol 1998;82:342-5.

27 Akita K, Kuga H. Digital processing of colour ocular fundus images. In: Linberg, Kaihara, ed. MEDINFO 80. Amsterdam: IFIP North-Holland Publishing Company, 1980:80-4.

28 Goldbaum MH, Karz NP, Nelson MR, et al. The discrimination of similarly coloured objects in computer images of the ocular fundus. Invest Ophthalmol Vis Sci 1990;31:617-23.

29 Schweitzer D, Hammer M, Scibor M. Imaging spectrometry in ophthalmology-principle and applications in microcirculation and in investigation of pigments. Ophthalmic Res 1996;28(suppl 2):37-44.

30 Ward NP, Tomilson S, Taylor CJ. Image analysis of fundus photographs: the detection and measurement of exudates associated with diabetic retinopathy. Ophthalmology 1989;96:80-6.

31 Kirkpatrick JNP. Spectral reflectance imaging of the ocular fundus using a scanning laser ophthalmoscope. MD thesis. University of Aberdeen, 1997.

32 Von Ruckmann A, Fitzke FW, Bird AC. Distribution of fundus autofluorescence with a scanning laser ophthalmoscope. Br F Ophthalmol 1995;79: 407-12.

33 Von Ruckmann A, Fitzke FW, Bird AC. In vivo fundus autofluorescence in macular dystrophies. Arch Ophthalmol 1997;115:609-15.

34 Webb RH, Hughes GW, Delori FC. Confocal scanning laser ophthalmoscope. Appl Opt 1987;26:1492-9
35 Zinser G, Harbarth U, Schroder. Formation and analysis of threedimensional data with the laser tomographic scanner (LTS). In: Nasemann JE, Burk ROW, eds. Scanning laser ophthalmoscopy and tomography. Munich: Quintessenz, 1990:243-52.

36 Hammer M, Roggan A, Schweitzer D, et al. Optical properties of ocular fundus tissues-an in vitro study using the double-integrating-sphere technique and inverse Monte Carlo simulation. Phys Med Biol 1995;40: 963-78.

37 Bartsch DU, Freeman WR. Laser-tissue interaction and artefacts in confocal scanning laser ophthalmoscopy and tomography. Neurosci Biobehav Rev 1993;17:459-67.

38 Bartsch DU, Freeman WR. Axial intensity distribution of the human retina with a confocal scanning laser tomograph. Exp Eye Res 1994;58:161-73.

39 Lachkar Y, Howard C. Reproducibility of optic nerve head topographic measurements with the Glaucoma-Scope. Eye 1997;11:810-7.

40 Broadway DC, Drance SM, Parfitt CM, et al. The ability of scanning laser ophthalmoscope to identify various glaucomatous optic disk appearances. Am 7 Ophthalmol 1998;125:593-604.

41 Orgul S, Croffi GA, Bacon DR, et al. Sources of variability of topometric data with a scanning laser ophthalmoscope. Arch Ophthalmol 1989;114: $161-4$.

42 Weinberger D, Stiebel-Kalish H, Gaton DD, et al. Three-dimensional measurements of topographical changes in macular diseases using confocal laser ophthalmoscope. Lasers Light Ophthalmol 1997;8:39-45.

43 Zambarakji HJ, Evans JE, Amoaku WMK, et al. Reproducibility of volumetric measurements of normal maculae with the Heidelberg retina tomography. Br F Ophthalmol 1998;82:884-91.

44 Vieira $\mathrm{P}$. Tomographic imaging with a scanning laser ophthalmoscope. $\mathrm{PhD}$ thesis. University of Aberdeen, 1999.

45 Jamsen K, Harju M. Optic disk tomography in patients with thyroid disease and optic neuropathy mimicking normal tension glaucoma. Ann Ophthalmol Glaucoma 1997;29:154-60.

46 Irak I, Zangwill L, Garden V, et al. Change in optic disk topography after trabeculectomy. Am f Ophthalmol 1996;122:690-5.

47 Beausencourt E, Elster AE, Hartnett ME, et al. Quantitative analysis of macular holes with scanning laser tomography. Ophthalmology 1997;104: 2018-29.

48 Ikeda T, Sato K, Katano T, et al. Examination of patients with cystoid macular oedema using scanning laser ophthalmoscope with infrared light. Am f Ophthalmol 1998;125:710-2.

49 Mulholland DA, Craig JJ, Rankin SJA. Use of scanning laser ophthalmoscopy to monitor papilloedema in idiopathic intracranial hypertension. $\mathrm{Br} \mathcal{F}$ Ophthalmol 1998;82:1301-5.

50 Hudson C, Flanagan JG, Turner GS, et al. Scanning laser tomography Z profile signal width as an objective index of macular retinal thickening. $\mathrm{Br} \mathcal{F}$ Ophthalmol 1998;82:121-30.

51 Dreher AW, Reiter KR. Retinal laser ellipsometry: a new method for measuring the retinal nerve fiber layer thickness distribution. Clin Vis Sci 1992; 7:481-8

52 Weinreb RN, Zangwill L, Berry CC, et al. Detection of glaucoma with scanning laser polarimetry. Arch Ophthalmol 1998;116:1583-9.

53 Poinoosawmy D, Fontana L, Wu JX, et al. Variation of nerve fiber layer thickness measurements with age and ethnicity by scanning laser polarimetry. Br F Ophthalmol 1997;81:350-4.

54 Anton A, Zangwill L, Emdadi A, et al. Nerve fiber layer measurements with scanning laser polarimetry in ocular hypertension. Arch Ophthalmol 1997;115:331-4

55 Zangwill L, Berry CA, Garden VS, et al. Reproducibility of retardation measurements with the nerve fiber analyser II. F Glaucoma 1997;6:384-9.

56 Junghardt A, Schmid MK, Schipper I, et al. Reproducibility of the data determined by scanning laser polarimetry. Graefes Arch Clin Exp Ophthalmol 1996;234:628-32.

57 Gaida G. Perspectives and limits of three-dimensional fundus microscopy. In: Nasemann JE, Burk ROW, eds. Scanning laser ophthalmoscopy and tomography. Munich: Quintessenz, 1990:253-7.

58 Van Kempen GMP, Van Vliet LJ, Verveer PJ, et al. A quantitative comparison of image restoration methods for confocal microscopy. $\mathcal{F}$ Microsc 1997; 185:354-65.

59 Ikeda T, Sato K, Katano T, et al. Examination of patients with cystoid macular edema using a scanning laser ophthalmoscope with infrared light. Am f Ophthalmol 1998;125:710-12.

60 Puliafito CA, Hee MR, Lin CP, et al. Imaging of macular diseases with optical coherence tomography. Ophthalmology 1995;102:217-29.

61 Baumann M, Gentile RC, Leibmann JM, et al. Reproducibility of retinal thickness measurements in normal eyes using optical coherence tomography. Ophthalmic Surg Lasers 1998;29:280-5.

62 Schuman JS, Pedut-Kloizman T, Hertzmark E, et al. Reproducibility of nerve fiber layer thickness measurements using optical coherence tomography. Ophthalmology 1996;103:1889-98.

63 Hee MR, Puliafito CA, Ducker JS, et al. Topography of diabetic macular oedema with optical coherence tomography. Ophthalmology 1998;105: 360-70

64 Roh S, Noecker RJ, Schuman JS, et al. Effect of optic nerve head drusen on nerve fiber layer thickness. Ophthalmology 1998;105:878-85.

65 Zangwill LM, Williams JM, Weinreb RN. Quantitative methods for evaluating the retinal nerve fiber layer in glaucoma. Ophthalmol Clin N Am 1998; 11:233-41.

66 Rutledge BK, Puliafito CA, Ducker JS, et al. Optical coherence tomography of macular lesions associated with optic nerve head pits. Ophthalmology 1996; 103:1047-53.

67 Hee MR, Baumal CR, Puliafito CA, et al. Optical coherence tomography of age-related macular degeneration and choroidal neovascularization. Ophthalmology 1996;103:1260-70. 